\title{
The Therapeutic Evaluation of Steroids in IgA Nephropathy Global (TESTING) Study: Trial Design and Baseline Characteristics
}

\author{
Muh Geot Wong a, b Jicheng Lv ${ }^{c, w}$ Michelle A. Hladunewich ${ }^{d}$ Vivekanand Jha ${ }^{e, f, g}$ \\ Lai Seong Hooi $^{\text {h Helen Monaghan }}{ }^{a}$ Minghui Zhao ${ }^{c}$ Sean Barbour ${ }^{i}$ Heather N. Reich ${ }^{j}$ \\ Daniel Cattran ${ }^{j}$ Richard Glassock ${ }^{k}$ Adeera Levin ${ }^{i}$ Meg J. Jardinel David C. Wheeler ${ }^{m}$ \\ Mark Woodward $^{\mathrm{a}, \mathrm{f}}$ Laurent Billot ${ }^{\mathrm{a}}$ Tak Mao Chan ${ }^{\mathrm{n}}$ Zhi-Hong Liu $^{\circ}$ David W. Johnson ${ }^{\mathrm{p}}$ \\ Alan Cass ${ }^{a, q}$ John Feehally ${ }^{r}$ Jürgen Floege ${ }^{s}$ Giuseppe Remuzzi ${ }^{t}$ Yangfeng Wu ${ }^{u}$ \\ Rajiv Agarwalv Hong Zhang ${ }^{c}$ Vlado Perkovic ${ }^{a}$ for the TESTING Study Group \\ aThe George Institute for Global Health, University of New South Wales, Sydney, NSW, Australia; ${ }^{b}$ Department of \\ Renal Medicine, Royal North Shore Hospital, St Leonards, NSW, Australia; 'Renal Division, Department of Medicine, \\ Peking University First Hospital, Beijing, China; ${ }^{d}$ Department of Medicine, Sunnybrook Health Sciences Centre, \\ University of Toronto, Toronto, ON, Canada; ${ }^{\mathrm{e} T h e}$ George Institute for Global Health India, New Delhi, India; \\ fThe George Institute for Global Health, School of Public Health, Imperial College, London, UK; 9Department of \\ Medicine, Prasanna School of Public Health, Manipal Academy of Higher Education, Manipal, India; ' ${ }^{\text {Department }}$ \\ of Medicine, Sultanah Aminah Hospital, Johor Bahru, Malaysia; 'Department of Medicine, The University of British \\ Columbia, Vancouver, BC, Canada; 'ivivision of Nephrology, University Health Network, Toronto, ON, Canada; \\ kDepartment of Medicine, David Geffen School of Medicine, University of California-Los Angeles, Los Angeles, \\ CA, USA; 'The NHMRC Clinical Trial Centres, University of Sydney, Camperdown, NSW, Australia; mepartment of \\ Renal Medicine, University College London, London, UK; "Department of Medicine, University of Hong Kong, Hong \\ Kong, China; ${ }^{\circ}$ National Clinical Research Center of Kidney Diseases, Jinling Hospital, Nanjing University School of

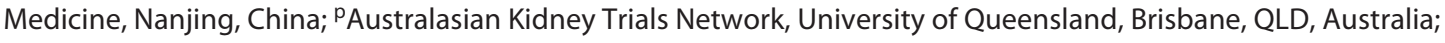 \\ 'Menzies School of Health Research, Charles Darwin University, Darwin, NT, Australia; 'Department of Renal

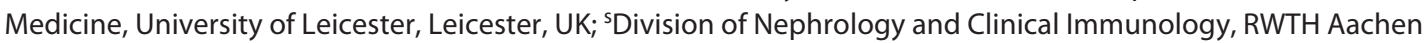

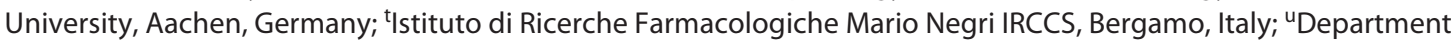 \\ of Epidemiology and Biostatistics, Peking University Clinical Research Institute, Beijing, China; vDepartment of

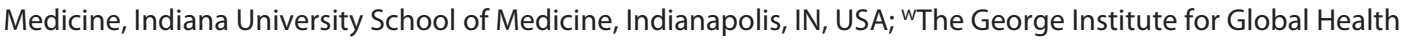 \\ China, Beijing, China
}

\section{Keywords}

$\lg$ A nephropathy · Glucocorticoids · Kidney failure

\begin{abstract}
Introduction: Despite optimal current care, up to $30 \%$ of individuals suffering from immunoglobulin A nephropathy (IgAN) will develop kidney failure requiring dialysis or kidney transplantation. The Therapeutic Evaluation of STe-
\end{abstract}

roids in IgA Nephropathy Global (TESTING) study was designed to assess the benefits and risks of steroids in people with IgAN. We report the trial design as well as the baseline characteristics of study participants. Methods: It is an investigator-initiated, multicenter, double-blind, placebocontrolled, randomized trial of individuals with kidney biopsy-confirmed IgAN, proteinuria $\geq 1 \mathrm{~g} /$ day, and an esti-

Trial Registration: Clinicaltrials.gov Identifier: NCT01560052.
C 2021 The Author(s).

Published by S. Karger AG, Basel

This is an Open Access article licensed under the Creative Commons Attribution-NonCommercial-4.0 International License (CC BY-NC) (http://www.karger.com/Services/OpenAccessLicense), applicable to the online version of the article only. Usage and distribution for commercial purposes requires written permission.
Correspondence to:

Muh Geot Wong, mwong@georgeinstitute.org.au

Hong Zhang, hongzh@bjmu.edu.cn 
mated GFR of $20-120 \mathrm{~mL} / \mathrm{min} / 1.73 \mathrm{~m}^{2}$, following at least 3 months of standard of care including maximum labelled (or tolerated) dose of renin-angiotensin system blockade. The original study design randomized participants $1: 1$ to oral methylprednisolone $(0.6-0.8 \mathrm{mg} / \mathrm{kg} /$ day, maximum 48 $\mathrm{mg} /$ day) for 2 months, with subsequent weaning by $8 \mathrm{mg} /$ day/month over 6-8 months, or matching placebo. The intervention was modified in 2016 (due to an excess of serious infection) to low-dose methylprednisolone $(0.4 \mathrm{mg} /$ $\mathrm{kg} /$ day, maximum $32 \mathrm{mg} /$ day) for 2 months, followed by weaning by $4 \mathrm{mg} /$ day/month over 6-9 months, or matching placebo. Participants recruited after 2016 also received prophylaxis against Pneumocystis jirovecii pneumonia during the first 12 weeks of treatment. Results: The study recruitment period extended from May 2012 to November 2019 . By the time the excess of serious infections was observed, 262 participants had been randomized to the original full-dose treatment algorithm, and an interim analysis was reported in 2016. Subsequently, 241 additional participants were randomized to a revised low-dose protocol, for a total of 503 participants from China (373), India (78), Canada (24), Australia (18), and Malaysia (10). The mean age of randomized participants was $38,39 \%$ were female, mean eGFR at randomization was $62.7 \mathrm{~mL} / \mathrm{min} / 1.73 \mathrm{~m}^{2}$, and mean 24-h urine protein $2.54 \mathrm{~g}$. The primary endpoint is a composite of $40 \%$ eGFR decline from baseline or kidney failure (dialysis, transplantation, or death due to kidney disease), and participants will be followed until the primary outcome has been observed in at least 160 randomized participants. Analyses will also be made across predefined subgroups. Effects on eGFR slope and albuminuria will also be assessed overall, as well as by the steroid dosing regimen. Conclusions: The TESTING study (combined full and low dose) will define the benefits of corticosteroid use on major kidney outcomes, as well as the risks of therapy, and provide data on the relative effects of different doses, in individuals with high-risk IgAN. @ 2021 The Author(s).

Published by S. Karger AG, Basel

\section{Introduction}

IgAN is the commonest cause of glomerulonephritis and the leading cause of kidney failure requiring dialysis in young adults worldwide with variable prevalence and regional differences in characteristics $[1,2]$. The natural history of IgAN is heterogeneous and varies from an indolent benign course to rapid progression to kidney failure requiring dialysis $[3,4]$. Proteinuria ( $>1$ g/day), hypertension, or elevated serum creatinine at the time of diagnosis [5, 6], as well as specific histological lesions on kidney biopsy (Oxford classification MEST-C scores) [79], have been identified as markers of poor prognosis. Despite improvements in the understanding of the pathological mechanisms of IgAN and improved disease-risk prediction, there is no current established disease-targeted therapy for individuals at high risk of kidney progression. Although corticosteroids and other immunosuppressants have been used for decades based on their biologically plausible effects on the course of IgAN, their role remains controversial $[10,11]$. An updated Cochrane review of immunosuppressive agents in IgAN that included 8 studies (741 participants) reported corticosteroid therapy may induce complete remission, prevent doubling of serum creatinine, and reduce proteinuria, but has uncertain effects on glomerular filtration rate, death, infection, and malignancy [12]. In the STOP-IgAN trial, addition of immunosuppression to supportive care in 162 Caucasian participants resulted in more adverse effects with little impact on eGFR decline over the 3-year trial period, or during a subsequently published 7 -year cohort follow-up $[13,14]$.

The Therapeutic Effects of STeroids in IgA Nephropathy Global (TESTING) study was designed to assess the effects of a 6- to 9-month course of oral steroids on the risk of major kidney outcomes, as well as the safety of this therapy. In 2015, TESTING was temporarily halted on the advice of the Data Safety Monitoring Committee (DSMC) due to a high rate of serious adverse events (primarily infections) among people randomized to steroid therapy. However, the results at that time also suggested potential benefits of corticosteroid use on kidney outcomes [15]. The trial design was subsequently modified and recruitment continued. Here, we report the final design of the TESTING study including the reported interim analysis and the protocol modifications, as well as the baseline characteristics of all randomized study participants.

\section{Methods}

Study Design and Participants

The Therapeutic Evaluation of STeroids in IgA Nephropathy Global (TESTING) study is an investigator-initiated, multicenter, double-blind, randomized controlled trial comparing the effects of oral methylprednisolone to matching placebo on the risk of major kidney outcomes in people with IgAN receiving appropriate supportive therapy, including maximum tolerated renin-angiotensin blocker (RAS) therapy.

The study is overseen by a Steering Committee, coordinated by an academic research organization (The George Institute for Glob- 


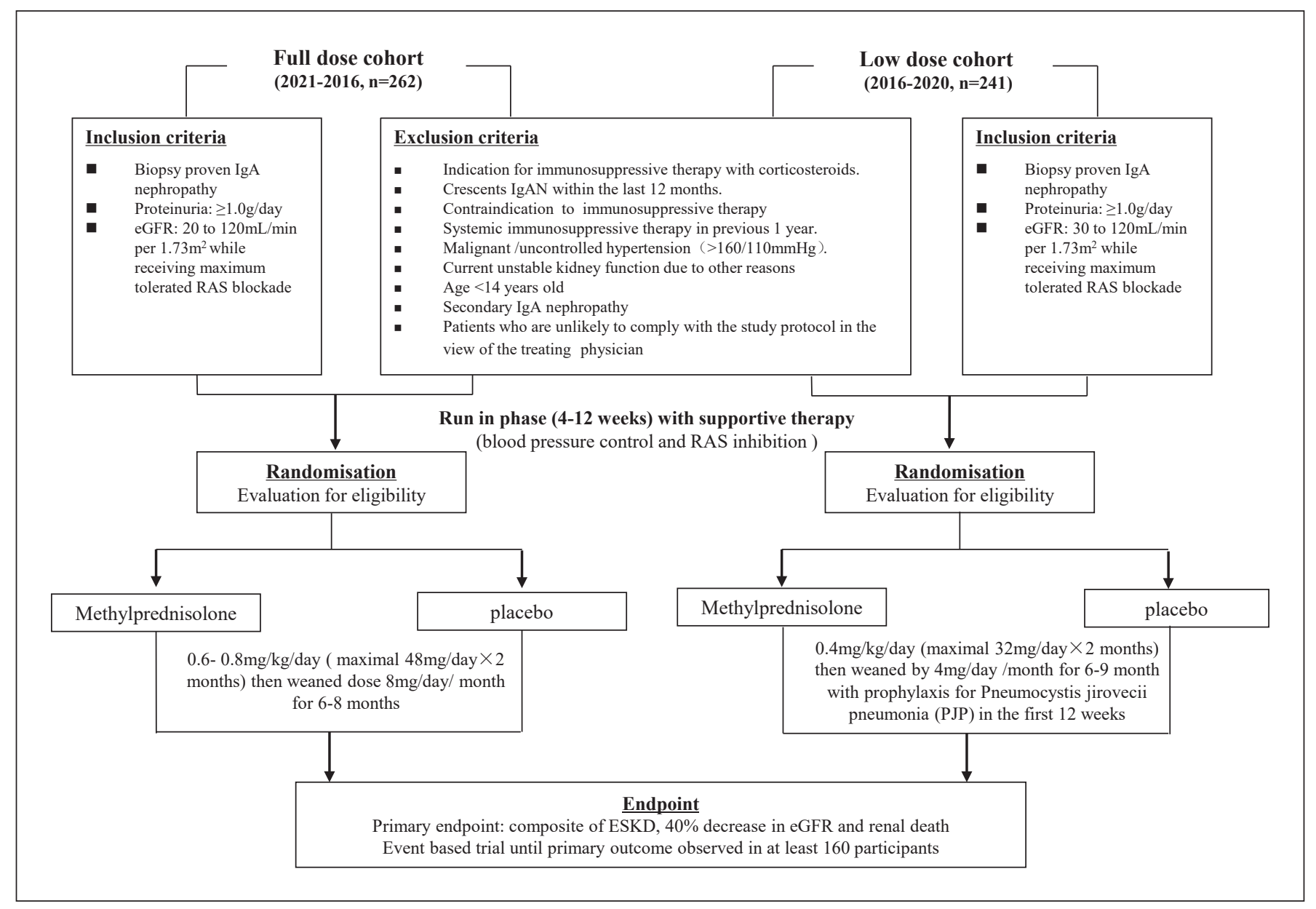

Fig. 1. Schematic diagram of the full corticosteroid and low corticosteroid dose cohort combined. PJP, Pneumocystis jirovecii pneumonia.

al Health), and conducted in sites in Australia, Canada, China including Hong Kong, India, and Malaysia. Ethical approval to conduct the trial was obtained from Peking University, the University of Sydney, and the ethics committees of individual countries and study sites, with all participants providing written informed consent. An independent Data Safety Monitoring Committee (DSMC) has been established and meets twice a year to review efficacy and safety data.

The study started recruitment in 2012 but was halted in November 2015 based on the advice of the DSMC due to safety concerns, primarily an excess of serious infections including 2 deaths in the steroid arm. An interim analysis of data collected to that time was published [15], and recruitment was recommenced following protocol modification to a reduced methylprednisolone dose regimen (see below), with ongoing follow-up of all participants (including the original cohort) with a revised sample size. Figure 1 shows a schematic diagram of the full steroid and low steroid dose cohort. A summary of the changes in the modified protocol is provided in online supplementary Table 1 (for all online suppl. material, see www.karger.com/doi/10.1159/000519812).

\section{Participants and Procedures}

Eligibility required a diagnosis of primary IgAN proven on kidney biopsy, an eGFR between 20 and $120 \mathrm{~mL} / \mathrm{min}$ per $1.73 \mathrm{~m}^{2}$ (calculated using the Chronic Kidney Disease Epidemiology Collaboration formula, modified to $30-120 \mathrm{~mL} / \mathrm{min}$ per $1.73 \mathrm{~m}^{2}$ for the low-dose cohort), and urinary protein excretion above $1 \mathrm{~g} /$ day. Exclusion criteria included a strong indication, or contraindication, to corticosteroid therapy based on the judgment of the treating physician, or the use of systemic immunosuppressive therapy in the previous year.

Potential participants who provided informed consent and met study entry criteria underwent a run-in period of 4-12 weeks to allow optimization of background therapy, including addition or titration to a maximum labelled or tolerated dose of RAS blockade as per standard of care. Participants who met the eligibility criteria at the end of the run-in period were randomly assigned 1:1 via a password-protected encrypted website interface to receive oral methylprednisolone or matching placebo, stratified by region, proteinuria $(<3 \mathrm{~g} /$ day or $\geq 3 \mathrm{~g} /$ day $)$, estimated GFR $(<50 \mathrm{~mL} /$ $\mathrm{min} / 1.73 \mathrm{~m}^{2}$ or $\geq 50 \mathrm{~mL} / \mathrm{min} / 1.73 \mathrm{~m}^{2}$ ), and kidney biopsy findings 
(endocapillary proliferation status according to the Oxford classification, E1 or E0). Randomization was performed using a minimization algorithm that was centrally generated and used by all sites to minimize any imbalances in key variables. Participants, investigators, site staff, trial committees, and sponsors were masked to treatment assignment for the duration of the study.

Participants were followed at regular intervals for a planned average follow-up period of around 5 years. Study assessments occurred monthly for 3 months after randomization, then every 3 months until month 12, and every 12 months until the end of this study. Additional telephone or face-to-face assessments were undertaken at 3-month intervals to ascertain kidney endpoints or adverse events. Laboratory evaluation of all specimens was performed in a national central laboratory in China and at local laboratories in other countries.

\section{Endpoints and Event Adjudication}

The primary composite endpoint is defined as the first occurrence of a sustained $40 \%$ decrease in eGFR, the development of kidney failure (defined as a need for maintenance dialysis or kidney transplantation and adjudicated by a blinded, independent committee), or death due to kidney disease. The $40 \%$ eGFR endpoint was chosen based on consensus recommendations from a workshop sponsored by the National Kidney Foundation and the US Food and Drug Administration on kidney endpoints and is now a well-accepted kidney endpoint for clinical trials [16-18]. Occurrences of $40 \%$ eGFR reduction are included as endpoints if they are confirmed on a repeat serum creatinine at least 30 days following their first occurrence, or if they occur at the final available measurement. Investigators are also asked to exclude reversible causes of eGFR decline including obstructive causes, nephrotoxins, IV contrast, and other causes for acute-on-chronic kidney failure.

Secondary endpoints include the composite of kidney failure and all-cause death with either $30 \%, 40 \%$, or $50 \%$ decrease in eGFR. The secondary endpoints also include the composite outcome of kidney failure and death due to kidney disease, individual components of the composite primary endpoint, proteinuria reduction evaluated by time-averaged proteinuria, and eGFR slope across all post-randomization study assessments.

A blinded event adjudication committee (EAC) consisting of experts in nephrology adjudicates kidney failure and deaths. For the purpose of event adjudication, kidney failure is defined as the necessity for maintenance dialysis (peritoneal or hemodialysis) for at least 90 days, or receipt of kidney transplantation. The 90 -day criterion is included in the definition of the kidney failure endpoint to avoid misclassification of kidney failure caused by acute kidney injury or volume overload requiring kidney replacement therapy. If kidney failure is reached $<90$ days before study closure or if the participant dies within 90 days of dialysis initiation, the EAC will adjudicate whether the endpoint meets kidney failure criteria, using the detailed definitions and criteria in the EAC charter.

Prespecified exploratory endpoints include (i) a 25\% decrease in eGFR assessed separately or as part of a composite with kidney failure and all-cause death, (ii) annual slope in the inverse of serum creatinine, (iii) proteinuria remission at 6 th, 12th, and 24th month, and (iv) hematuria disappearance at the last follow-up. A statistical analysis plan will be finalized and locked prior to data unblinding.

\section{Adverse Events and Safety}

Predefined safety outcomes are all-cause mortality, total serious adverse events, serious infections, new diabetes, gastrointestinal hemorrhage, fracture or osteonecrosis, and cardiovascular events. Serious adverse events are defined according to the International Conference on Harmonization Guideline for Clinical Safety Data Management.

\section{Intervention}

In the original study design, participants were randomized 1:1 to oral methylprednisolone $(0.6-0.8 \mathrm{mg} / \mathrm{kg} / \mathrm{day}$, maximum $48 \mathrm{mg} /$ day) or matching placebo for 2 months, with subsequent weaning by $8 \mathrm{mg}$ /day over $6-8$ months. Protocol modifications were made to incorporate measures to reduce infection risk following published interim analysis results [15]. The steroid dose was reduced to methylprednisolone $0.4 \mathrm{mg} / \mathrm{kg} /$ day or matching placebo (maximum $32 \mathrm{mg}$ /day) for 2 months, followed by dose tapering by 4 mg/day/month over 6-9 months. Prophylaxis for Pneumocystis jirovecii pneumonia was also implemented during the first 12 weeks of the treatment period for all subsequent participants.

\section{TESTING Substudies}

Two TESTING substudies are also planned. These are prospectively defined. The first aims to evaluate the histopathology of kidney biopsies by a central pathology committee using a standardized scoring system to validate the MEST-C Oxford classification scoring system [9] and to correlate the impact of pathology on steroid treatment response as well as short- and long-term outcomes for individuals with IgAN. The second substudy will serve as an external validation of the International IgA Nephropathy Prediction Model by the International IgAN Network research group collaboration, to predict proteinuria reduction following corticosteroid treatment [19].

\section{Statistical Analysis}

The initial sample size calculations assumed an annual combined event rate for the primary endpoint (50\% eGFR decrease, kidney failure, and death due to kidney disease) of $7 \%$, requiring 1,300 participants to detect a $30 \%$ relative risk reduction in the primary endpoint with $90 \%$ power $(\alpha=0.05)[20,21]$. Subsequent to the safety concerns and analysis and using a $40 \%$ eGFR reduction threshold rather than the original 50\% reduction, the sample size was revised to 500 participants in order to provide $90 \%$ power ( $\alpha$ $=0.05$ ) to detect an overall $40 \%$ risk reduction with a corticosteroid-based treatment after an average follow-up of 5 years across the combined (original and low dose) cohorts. These calculations assumed annual event rates of $40 \%$ decline in eGFR or kidney failure of $12 \%$ in the placebo arm and allowing for up to $10 \%$ of participants being lost to follow-up or having outcome data unavailable. The increase in the detectable treatment effect from a $30 \%$ to a $40 \%$ relative risk reduction was a pragmatic decision based on a balance between feasibility, funding, and plausible benefit given that the interim analysis results suggested the effect size may be as large as $63 \%$ [15].

The trial is event driven and has been designed to continue until at least 160 primary endpoints have been observed with an expected overall mean follow-up of 5 years. All analyses will be based on the intention-to-treat principle for all randomized participants, with every effort made to ensure complete follow-up data are available. 
Table 1. Baseline characteristic of TESTING participants

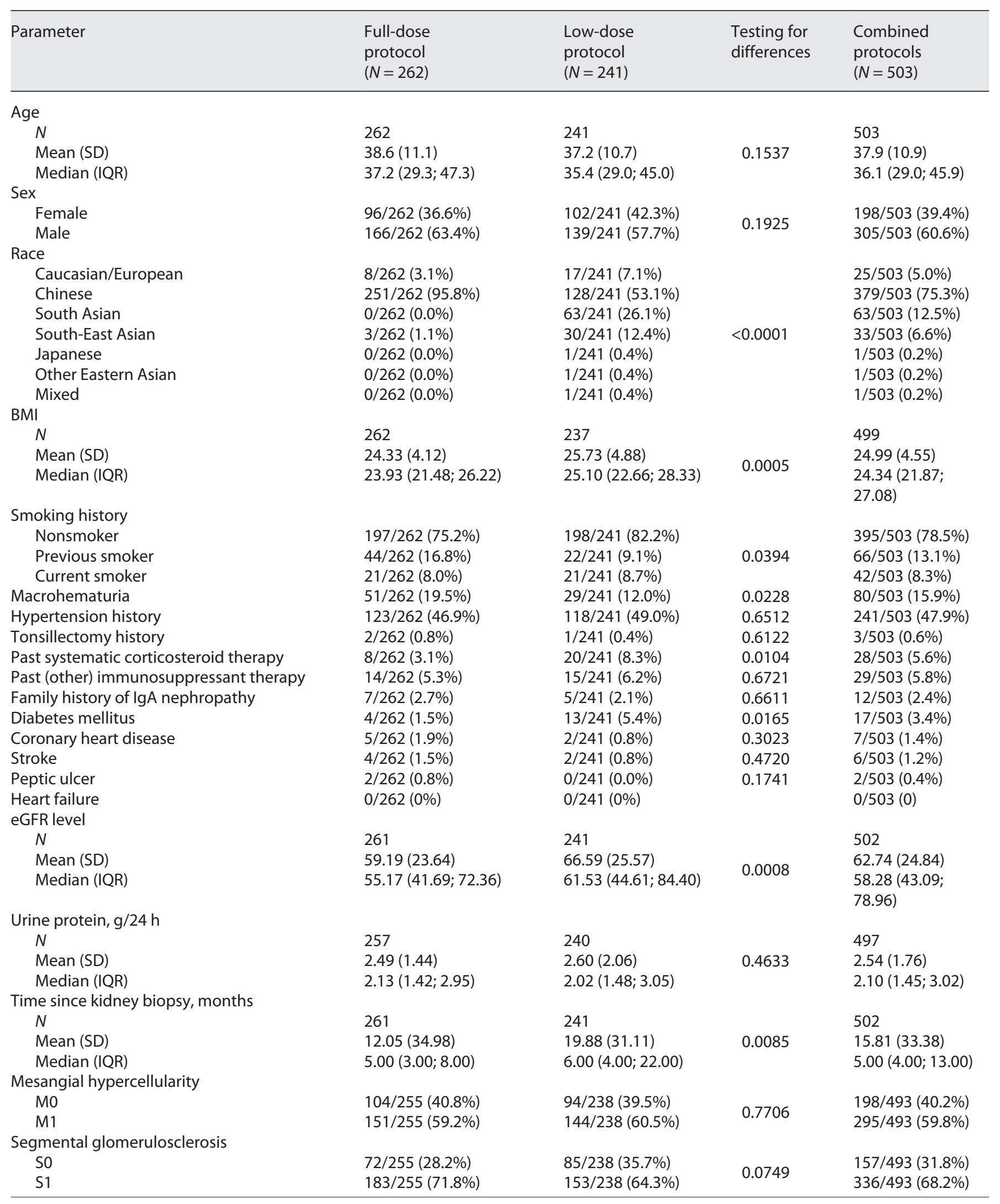


Table 1 (continued)

\begin{tabular}{|c|c|c|c|c|}
\hline Parameter & $\begin{array}{l}\text { Full-dose } \\
\text { protocol } \\
(N=262)\end{array}$ & $\begin{array}{l}\text { Low-dose } \\
\text { protocol } \\
(N=241)\end{array}$ & $\begin{array}{l}\text { Testing for } \\
\text { differences }\end{array}$ & $\begin{array}{l}\text { Combined } \\
\text { protocols } \\
(N=503)\end{array}$ \\
\hline \multicolumn{5}{|c|}{ Endocapillary hypercellularity } \\
\hline E0 & 189/262 (72.1\%) & 187/241 (77.6\%) & \multirow{2}{*}{0.1594} & $376 / 503(74.8 \%)$ \\
\hline E1 & $73 / 262(27.9 \%)$ & $54 / 241(22.4 \%)$ & & $127 / 503(25.2 \%)$ \\
\hline \multicolumn{5}{|c|}{ Tubular atrophy/interstitial fibrosis } \\
\hline T0 & $94 / 255$ (36.9\%) & $147 / 238(61.8 \%)$ & \multirow{3}{*}{$<0.0001$} & $241 / 493(48.9 \%)$ \\
\hline T1 & $118 / 255(46.3 \%)$ & $69 / 238(29.0 \%)$ & & $187 / 493(37.9 \%)$ \\
\hline $\mathrm{T} 2$ & $43 / 255(16.9 \%)$ & $22 / 238(9.2 \%)$ & & $65 / 493(13.2 \%)$ \\
\hline
\end{tabular}

Survival curves and estimated median survival times will be generated according to the Kaplan-Meier method and compared using the log-rank test. Cox proportional hazards will be used to estimate the hazard ratio comparing the 2 intervention groups. Analyses will be censored at the date when participants died (for causes other than kidney disease), were lost to follow-up, withdrew from study, or at the end of trial assessment, whichever occurred first. The Cox model will include the stratification variables (region, proteinuria, eGFR, and kidney biopsy findings) as fixed covariates. All $p$ values will be 2 -sided, and $p$ values $<0.05$ will be considered to indicate statistical significance. All analyses will be performed using the SAS software, version 9.4 (SAS Institute). A detailed analysis plan has been developed and is available as a preprint (https://doi.org/10.31219/osf.io/d7qrw).

\section{Subgroup Analyses}

The homogeneity of the treatment effects on the primary outcome across specified subgroups will be tested by adding interaction terms to the Cox models, with predefined subgroups including steroid dosage regimen (original protocol or reduced dose), baseline proteinuria ( $<3.0 \mathrm{vs}$. $\geq 3.0 \mathrm{~g} /$ day), baseline estimated GFR ( $<50$ vs. $\geq 50 \mathrm{~mL} / \mathrm{min}$ per $1.73 \mathrm{~m}^{2}$ ), endocapillary hypercellularity on histological scoring (yes or no), and race (Asian vs. non-Asian). As we believe the subgroup analysis by the steroid dosing regimen will be important, although power will be limited to assess heterogeneity for the primary outcome, we propose to focus on the subgroup analyses of effects on proteinuria and on eGFR slope if an overall benefit is observed. Given the limited power to detect interactions, for the dose subgroup (full dose vs. lower dose), differences in effect sizes will take precedence over the interaction $p$ value in guiding the interpretation and potential clinical significance.

\section{TESTING Steering Committee and Funding Source}

TESTING is an investigator-initiated trial funded by Peking University First Hospital, China, for all China sites; CIHR grant MOP126078 for sites in Canada, and NHRMC Project grant APP1042474 for central co-ordination and the remaining sites in the other participating countries. The study is overseen by a Steering Committee that designed the study, oversees the conduct of the trial, and will undertake the analysis and reporting of all data. All authors will have access to study results. Study drug and some initial seed funding were provided by Pfizer who have no other role in the TESTING trial. Matching placebo is manufactured locally in China or Australia, packaged and labelled, and distributed to the sites.

\section{Results}

Between May 2012 and November 2019, a total of 950 potentially eligible participants were screened, of whom 503 (58.6\%) were eligible and underwent randomization. This includes 523 potentially eligible participants who were screened for the original full-dose steroid regimen, of whom $262(48.8 \%)$ were eligible and underwent randomization, as well as 427 potentially eligible participants who were screened for the low-dose protocol, of whom $241(61.0 \%)$ were randomized. Among the randomized participants, 373 (73.7\%) were from China including Hong Kong, 78 (15.5\%) from India, 24 (4.7\%) from Canada, 18 (3.5\%) from Australia, and $10(1.9 \%)$ from Malaysia (Fig. 1). The complete list of participating sites is provided in online supplementary Table 2.

The baseline characteristics of the randomized participants overall and by steroid treatment regimen (high and low dose) are shown in Table 1. The mean age was $37.9 \pm$ 10.9 years and $60.6 \%$ were male. A majority $(75.3 \%)$ of the participants were Han Chinese, with smaller proportions of South Asian (12.5\%) and Caucasian (5.0\%) ethnicity. A history of macroscopic hematuria was reported by $15.9 \%$ of participants and $2.4 \%$ reported a family history of IgA nephropathy. Nearly half (47.9\%) had a history of hypertension, and other comorbidities were infrequent. At baseline, 53.3\% were taking an angiotensin-converting enzyme inhibitor and $47.5 \%$ an angiotensin receptor blocker. Combined use of angiotensin-converting enzyme inhibitors and angiotensin receptor blockers is pro- 
hibited by the protocol to minimize the risk of hyperkalemia and/or acute kidney injury.

The mean eGFR at baseline was $62.7 \pm 24.8 \mathrm{~mL} /$ $\min / 1.73 \mathrm{~m}^{2}$, and the mean 24 -h urine protein excretion was $2.5 \pm 1.8 \mathrm{~g} /$ day. One quarter of the participants had evidence of endocapillary hypercellularity on kidney biopsy, while about half had evidence of fibrosis and twothirds had segmental glomerulosclerosis.

Participants randomized to the full-dose compared to the low-dose steroid therapy regimen have broadly similar characteristics. The mean eGFR of the low-dose cohort was higher (66.6 vs. $59.2 \mathrm{~mL} / \mathrm{min} / 1.73 \mathrm{~m}^{2}, p<0.001$ ), but proteinuria levels ( 2.5 vs. $2.6 \mathrm{~g} /$ day), baseline comorbidities, and biopsy findings were broadly comparable.

\section{Discussion}

TESTING is the largest randomized trial conducted in IgA nephropathy to date, having successfully randomized 503 participants, and will determine the long-term efficacy and safety of oral methylprednisolone compared to matching placebo, on a background of RAS inhibitor therapy, in high-risk participants with IgA nephropathy. Although it is clear that high doses of immunosuppressants, such as corticosteroids, carry significant side effects, corticosteroid therapy in high-risk individuals with IgA nephropathy remains an area of great interest and some controversy, largely driven by uncertainty about its role in eGFR preservation and long-term protection against kidney failure.

The uncertainty about kidney benefits of corticosteroid-based immunosuppression is based on inconsistency in the available clinical trial-based outcome data. The STOP IgA trial compared steroid-based immunosuppression to placebo in high-risk but well-treated participants with IgAN and did not show any difference between groups in its eGFR-based primary outcome of decline of at least $15 \mathrm{~mL} / \mathrm{min} / 1.73 \mathrm{~m}^{2}$ over time [13]. In contrast, the interim analyses reported at the time of the change in protocol for the TESTING study suggested that the corticosteroid-treated group may have had a lower risk of a $40 \%$ decline in eGFR, kidney failure, or death due to kidney disease (5.9 vs. $15.9 \%$, HR 0.37 , 95\% CI: $0.17-$ $0.85, p=0.019$ ) and a slower rate of eGFR decline over time [15]. In this context, the additional data that will be provided by the final analysis of the TESTING study will be critical. They will provide increased power to detect reductions in the risk of a composite kidney outcome routinely accepted by regulators thus allowing the detec- tion of a smaller overall treatment effect ( $40 \%$ risk reduction) than that suggested by the previously published effects (63\% risk reduction).

It will also provide much longer follow-up than the previously reported trials, thereby providing critical information about the timing and long-term durability of any observed effects. The clinical significance of followup duration in IgAN is exemplified by previous studies in people with sustained proteinuria between 1 and $2 \mathrm{~g} /$ day where the 10 -year risk of $50 \%$ eGFR decline or kidney failure was $24 \%$ [22]. However, the short-term risk of similar clinical outcomes was only $1.1 \%$ in 3 years [22, 23].

The TESTING study will also provide key guidance about the safety of steroid-based regimens when used in IgA nephropathy. The previously reported interim data found a significantly increased risk of serious adverse events in the corticosteroid group ( 14.7 vs. $3.2 \%, p=003$ ) including 11 serious infections, 2 of which were fatal. The nearly 5 times higher risk of serious adverse events is a particularly important finding given that many previous corticosteroid trials in IgAN have collected and reported adverse effects inconsistently. The STOP-IgAN trial also reported a similarly high incidence of severe infections among the immunosuppression arm ( 8 vs. $3, p=0.07$, compared to the control groups), including 1 death in the combination immunosuppression group. The excess of serious adverse events (mostly infections) observed in recent studies has changed clinical decision-making as indicated by the most recent KDIGO guidelines in glomerulonephritis for the use of corticosteroids in IgAN. The KDIGO guidelines in glomerulonephritis 2020 recommend a 6-month course of steroid therapy in individuals with high-risk IgAN and advised caution particularly in those who have an eGFR below $50 \mathrm{~mL} / \mathrm{min} / 1.73 \mathrm{~m}^{2}$ (currently under public review). The final TESTING study will provide important data that will help resolve the uncertainty. In addition to the previously reported risk of serious adverse events and infections with the full-dose steroid regimen, the trial will allow at least qualitative comparison of infection rates with a lower-dose regimen accompanied by routine antibiotic prophylaxis against $P$. jirovecii. These data will allow clinicians to better balance any potential benefits against the risks associated with different regimens and help support and guide decisionmaking for people with IgA nephropathy.

The TESTING study will allow some comparison of any observed benefits of steroid therapy between the full-dose and low-dose regimens. As the primary composite outcome events are heavily time dependent and the follow-up dura- 
tion of participants randomized to full-dose steroids or placebo will be substantially longer than those randomized to low-dose steroids or placebo, the majority of the primary outcomes is expected to occur in the former group. As such, it is unlikely that the TESTING study will have sufficient statistical power to reliably assess interactions by dose; however, it should provide important insights into the effects of different steroid therapy on proteinuria reduction and rate of eGFR loss. While the importance of proteinuria reduction continues to be debated, subject-level analyses of data from 13 randomized controlled trials found that shortterm reductions in proteinuria predict meaningful kidney clinical outcomes (i.e., doubling of serum creatinine level, kidney failure, and/or death). These analyses support the use of proteinuria reduction as a surrogate endpoint in IgA nephropathy trials [24], and regulatory agencies are considering interim approval of new therapies based on demonstrated proteinuria reduction in this condition (https:// www.calliditas.se/en/interim-report-q1-2021-3530/).

The relationship between rate of eGFR loss and change in proteinuria appears to be even more strongly related to kidney benefits [25]. As a result, any differences in effects on proteinuria reduction or rate of eGFR loss are likely to represent real differences in any kidney-protective effects of steroid therapy.

Despite the promising nature of the reported NEFIGAN [26] and NEFIGARD (https://www.calliditas.se/en/ interim-report-q1-2021-3530/) results and several ongoing trials with promising novel agents in IgA nephropathy, the outcomes of the TESTING study remain relevant because if steroids are shown to be beneficial with acceptable risk, it would have immediate implications globally, but especially for low-resource countries - given the relatively low cost of the intervention as compared to the new agents which are likely to be substantially more expensive and less accessible for these countries.

A major challenge to the trial design was the early observation of significant harm associated with full-dose steroid therapy. Following the DSMC report, the TESTING Steering Committee made changes designed to protect study participants while maximizing the value of the trial data that had been collected and could be collected in the future. All participants still receiving study drug were unblinded and were asked to return for an additional study assessment at which they were informed about the results that had been observed, and updated informed consent for ongoing data collection, safety assessment, and additional information regarding study outcomes was obtained. The study was then transitioned to an ongoing follow-up phase for all participants randomized to that time, recognizing that the original study design required up to 8 months of steroid/placebo treatment, but up to 5 years of average follow-up. Based on the data analyses undertaken at the time to allow actual and potential study participants and their treating clinicians to be fully informed, with observed results that suggest a potential benefit of corticosteroid in high-risk individuals, the Steering Committee made the decision to continue recruitment with a protocol amendment instituting safety strategy. The safety strategy put in place included a lower-dose treatment regimen for subsequently randomized participants as well as Pneumocystis jirovecii pneumonia prophylaxis with trimethoprim-sulfamethoxazole during the first 3 months. With these modifications, along with changes to the study design, recruitment to the TESTING trial was successfully completed.

Furthermore, the trial population appeared to be comparable across the 2 cohorts, supporting cautious comparison of treatment effects across the 2 groups. The observed differences between the baseline characteristics of the high-dose and low-dose TESTING cohorts in BMI, smoking history, corticosteroid use, and history of diabetes mellitus may reflect the greater numbers of predominantly Caucasian (Canada) and East Asian (India) population in the low-dose cohort. The inclusion criteria of eGFR $>30 \mathrm{~mL} / \mathrm{min} / 1.73 \mathrm{~m}^{2}$ explain for the baseline eGFR and tubulointerstitial fibrosis scores. There is comparable baseline proteinuria and other comorbidities between cohorts. The final study plan was designed to maximize the information provided to people with IgA nephropathy and the broader kidney community, in order to support data-driven treatment decisions in the future.

\section{Conclusion}

Significant uncertainty persists in the role of steroid therapy in the treatment of IgAN. The TESTING trial will (1) confirm whether corticosteroid therapy produces long-term benefits overall, (2) provide important data by dose regimen on major kidney outcomes in a multiethnic population, and (3) allow this to be weighed against shortterm complications of therapy. This will allow better informed decision-making for people with high-risk IgA nephropathy in the future.

\section{Acknowledgments}

The authors would like to acknowledge the contribution of the following individuals: TESTING Data Safety and Monitoring Committee: David Jayne (Chair), Angela Yee-Moon Wang, Tom Greene, 
and Michael Walsh; Endpoint adjudication committee: Rajiv Aggarwal (Chair), Zou Li, Amanda Wang, and Amanda Mather; key coordinating center staff of each country: Rebecca Anderson (Australia); Ainy Zahid and Anna Rogowsky (Canada); Yunfei Bao and Guili Sui (China); Oommen John and Purnima Rao Jevaji (India); Lee Mei Tan and Rozilawati Abdul Gaffar (Malaysia); George Clinical staff in Australia, China, India, and Malaysia; Data management team: Manuela Armenis, Dominic Byrne, and Ravinder Singh; Statistical team: Kris Rogers, Sana Shan, and Sandrine Stepien.

\section{Statement of Ethics}

The research described was conducted ethically in accordance with the World Medical Association Declaration of Helsinki. As noted in the manuscript, subjects gave their written informed consent to participate, and ethical approval was received from the respective Human Research Ethics Committee of each site of the individual country.

\section{Conflict of Interest Statement}

Muh Geot Wong has received fees for advisory boards, steering committee roles, or scientific presentations from Travere, Baxter, Amgen, Abbvie, Chinook, Dimerix, Otsuka, GlaxoSmithKline, and CSL-Behring. JiChing Lv has received consultation fees from Chinook therapeutics and KBP Biosciences. Michelle A. Hladunewich has received research funding from Chemocentryx, Ionis, Calliditas Therapeutics, Pfizer, and Roche and consultation fees from Alnylam Pharmaceuticals. Vivekanand Jha has received research grants and honoraria for consultancy and advisory board from Baxter, GSK, and AstraZeneca. Minghui Zhao has received consultation fees from Novartis, Roche, AstraZeneca, and GlaxoSmithKline. David Johnson has received consultancy fees, research grants, speaker's honoraria, and travel sponsorships from Baxter Healthcare and Fresenius Medical Care, consultancy fees from AstraZeneca, Bayer, and AWAK, speaker's honoraria from Ono and Boehringer Ingelheim and Lilly, and travel sponsorships from Ono and Amgen. David Johnson is the current recipient of an Australian National Health and Medical Research Council (NHMRC) Leadership Investigator Grant. Laurent Billot has received speaker's honoraria from Novo Nordisk. Heather Reich has received consultation honoraria from Calliditas, Chinook, Omeros, Alnylam, Retrophin, and Norvartis. Meg J. Jardine is supported by a Medical Research Future Fund Next Generation Clinical Researchers Program Career Development Fellowship; is responsible for research projects that have received unrestricted funding from Amgen, Baxter, CSL, Eli Lilly, Gambro, and MSD; has served on advisory boards sponsored by Akebia, AstraZeneca, Baxter, Bayer, Boehringer Ingelheim, MSD, and Vifor; serves/has served on Steering Committee for trials sponsored by Chinook, CSL, and Janssen; serves on a Steering Committee for an investigator-initiated trial with funding support from Dimerix; spoken at scientific meetings sponsored by Amgen, Janssen, Merck, Roche, and Vifor, with any consultancy, honoraria, or travel support paid to her institution. Mark Woodward has received consultation fees from Amgen, Kyowa Kirin, and Freeline. David C. Wheeler has received honoraria and/or speaker fees from AstraZeneca, Astel- las, Amgen, Bayer, Boehringer Ingelheim, GlaxoSmithKline, Janssen, Napp, Mundipharma, Merck Sharp and Dohme, Tricia, Vifor, and Zydus. Richard Glassock has received consultation fees from Chemocentryx, Omeros, Ionis, Travere, Horizon, BioCryst, Equillium, Calliditas, RenaSight (Natera), Novartis, Otsuka, and UpTo Date (Wolters Kluwer Health). Alan Cass was a recipient of the Australian NHMRC Project Grant for the current study and a current recipient of a NHMRC Leadership Investigator Grant. Jürgen Floege has received consultation honoraria from Abgenix, Calliditas, Idorsia, Novartis, Omeros, Travere, and Visterra. Giuseppe Remuzzi has received consultation fees from BioCryst Pharmaceuticals, Inc., and Menarini Ricerche Spa. Yangfeng Wu has received consultation fees from the World Health Organization, China Office, for the hypertension and noncommunicable disease program. Rajiv Agarwal has received consultation fees from Akebia, Boehringer, Bayer, Chinook, Diamedica, Reata, Vifor, and Vertex. Hong Zhang has received consultation fees and serves in the Steering Committee for Janssen, Novartis. Omeros, Calliditas, and Chinook. Vlado Perkovic has received fees for advisory boards, steering committee roles, or scientific presentations from AbbVie, Astellas, AstraZeneca, Bayer, Baxter, Bristol-Myers Squibb, Boehringer Ingelheim, Chinook, Dimerix, Durect, Eli Lilly, Gilead, GlaxoSmithKline, Janssen, Merck, Mitsubishi Tanabe, Mundipharma, Novartis, Novo Nordisk, Pfizer, PharmaLink, Relypsa, Sanofi, Servier, Travere, Vifor, and Tricida.

\section{Funding Sources}

The TESTING trial was funded by the Australian National Health and Medical Research Council, China CMA Innovation fund for Medical Science (2019-I2M-5-046), China National Funds for Distinguished Young Scientists (81925006), National Key Research and Development of Program of China (2018YFC1314004), the Canadian Institutes for Health Research, and had study drug and a small amount of initial funding (US\$75,000) provided by Pfizer. The funder of the study had no role in study design, data collection, data analysis, data interpretation, or writing of the report. The steering committee had final responsibility for the decision to submit for publication.

\section{Author Contributions}

Muh Geot Wong and Ji Cheng Lv were responsible for drafting the manuscript; Hong Zhang and Vlado Perkovic were responsible for providing critical input. All co-authors have equal contribution to the design and conduct of the study and provided the final approval for publication.

\section{Data Availability Statement}

The data that support the findings of this study are not publicly available because they contain information that could potentially compromise the privacy of the research participants and are however available from the corresponding author Muh Geot Wong and/or Hong Zhang upon reasonable request. 


\section{References}

1 D'Amico G. The commonest glomerulonephritis in the world: IgA nephropathy. Q J Med. 1987;64(245):709-27.

2 Schena FP, Pesce F. Epidemiology and ancestral difference. Chapter 2: epidemiology and ancestral difference. Singapore World Scientific; 2009. p. 9-19.

3 Kim JK, Kim JH, Lee SC, Kang EW, Chang TI, Moon SJ, et al. Clinical features and outcomes of IgA nephropathy with nephrotic syndrome. Clin J Am Soc Nephrol. 2012;7(3): $427-36$.

4 Donadio JV, Bergstralh EJ, Grande JP, Rademcher DM. Proteinuria patterns and their association with subsequent end-stage renal disease in IgA nephropathy. Nephrol Dial Transplant. 2002;17(10):1197-203.

5 Reich HN, Troyanov S, Scholey JW, Cattran DC; for the Toronto Glomerulonephritis Registry. Remission of proteinuria improves prognosis in IgA nephropathy. J Am Soc Nephrol. 2007;18(12):3177-83.

6 Liu L, Yin Z, Ma J, Duan S, Chen X. Potential association of body constitution with the prognosis of IgA nephropathy: a long-time follow-up of 203 cases in China. Evid Based Complement Alternat Med. 2019;2019: 6289478.

7 Working Group of the International IgA NN, the Renal Pathology Society, Cattran DC, Coppo R, Cook HT, Feehally J, et al. The Oxford classification of IgA nephropathy: rationale, clinicopathological correlations, and classification. Kidney Int. 2009;76(5):534-45.

8 Haas M, Verhave JC, Liu ZH, Alpers CE, Barratt J, Becker JU, et al. A Multicenter Study of the predictive value of cescents in IgA nephropathy. J Am Soc Nephrol. 2017;28(2): 691-701.

9 Trimarchi H, Barratt J, Cattran DC, Cook HT, Coppo R, Haas M, et al. Oxford classification of IgA nephropathy 2016: an update from the IgA Nephropathy Classification Working Group. Kidney Int. 2017;91(5):1014-21.

10 Floege J, Amann K. Primary glomerulonephritides. Lancet. 2016;387(10032):2036-48.
11 Lv J, Xu D, Perkovic V, Ma X, Johnson DW, Woodward M, et al. Corticosteroid therapy in IgA nephropathy. J Am Soc Nephrol. 2012; 23(6):1108-16.

12 Natale P, Palmer SC, Ruospo M, Saglimbene VM, Craig JC, Vecchio M, et al. Immunosuppressive agents for treating IgA nephropathy. Cochrane Database Syst Rev. 2020;2020(3): CD003965.

13 Rauen T, Eitner F, Fitzner C, Sommerer C, Zeier M, Otte B, et al. Intensive supportive care plus immunosuppression in IgA nephropathy. N Engl J Med. 2015;373(23): 2225-36.

14 Rauen T, Wied S, Fitzner C, Eitner F, Sommerer C, Zeier M, et al. After ten years of follow-up, no difference between supportive care plus immunosuppression and supportive care alone in IgA nephropathy. Kidney Int. 2020;98(4):1044-52.

15 Lv J, Zhang H, Wong MG, Jardine MJ, Hladunewich $\mathrm{M}$, Jha $\mathrm{V}$, et al. Effect of oral methylprednisolone on clinical outcomes in patients with IgA nephropathy: the TESTING randomized clinical trial. JAMA. 2017;318(5): 432-42.

16 Coresh J, Turin TC, Matsushita K, Sang Y, Ballew SH, Appel LJ, et al. Decline in estimated glomerular filtration rate and subsequent risk of end-stage renal disease and mortality. JAMA. 2014;311(24):2518-31.

17 Levey AS, Inker LA, Matsushita K, Greene T, Willis $\mathrm{K}$, Lewis $\mathrm{E}$, et al. GFR decline as an end point for clinical trials in CKD: a scientific workshop sponsored by the national kidney foundation and the US food and drug administration. Am J Kidney Dis. 2014;64(6):82135.

18 Levin A, Agarwal R, Herrington WG, Heerspink HL, Mann JFE, Shahinfar S, et al. International consensus definitions of clinical trial outcomes for kidney failure: 2020. Kidney Int. 2020;98(4):849-59.
19 Barbour SJ, Coppo R, Zhang H, Liu ZH, Suzuki Y, Matsuzaki K, et al. Evaluating a new international risk-prediction tool in IgA nephropathy. JAMA Intern Med. 2019;179(7): 942-52.

20 Li X, Liu Y, Lv J, Shi S, Liu L, Chen Y, et al Progression of IgA nephropathy under current therapy regimen in a Chinese population. Clin J Am Soc Nephrol. 2014;9(3):484-9.

21 Manno C, Torres DD, Rossini M, Pesce F, Schena FP. Randomized controlled clinical trial of corticosteroids plus ACE-inhibitors with long-term follow-up in proteinuric IgA nephropathy. Nephrol Dial Transplant. 2009; 24(12):3694-701.

22 Barbour SJ, Espino-Hernandez G, Reich HN, Coppo R, Roberts IS, Feehally J, et al. The MEST score provides earlier risk prediction in lgA nephropathy. Kidney Int. 2016;89(1): 167-75.

23 Barbour S, Feehally J. An update on the treatment of IgA nephropathy. Curr Opin Nephrol Hypertens. 2017;26(4):319-26.

24 Thompson A, Carroll K, A Inker L, Floege J, Perkovic V, Boyer-Suavet S, et al. Proteinuria reduction as a surrogate end point in trials of IgA nephropathy. Clin J Am Soc Nephrol. 2019;14(3):469-81.

25 Levey AS, Gansevoort RT, Coresh J, Inker LA, Heerspink HL, Grams ME, et al. Change in albuminuria and GFR as end points for clinical trials in early stages of CKD: a scientific workshop sponsored by the national kidney foundation in collaboration with the US food and drug administration and european medicines agency. Am J Kidney Dis. 2020;75(1): 84-104.

26 Fellstrom BC, Barratt J, Cook H, Coppo R, Feehally J, de Fijter JW, et al. Targeted-release budesonide versus placebo in patients with IgA nephropathy (NEFIGAN): a doubleblind, randomised, placebo-controlled phase 2b trial. Lancet. 2017;389(10084):2117-27. 